\title{
EJACULAÇÃO INDUZIDA EM TOURO (BOS TAURUS INDICUS) POR ESTIMULAÇÃO MANUAL DO PÊNIS
}

\author{
Bull (Bos taurus indicus) ejaculation induced by manual stimulation of the penis
}

Romildo Romualdo Weiss ${ }^{1},{ }^{*}$ Ana Claudia Machinski Rangel Abreu", Eduarda Maciel Busato ${ }^{1}$, Tácia Gomes Bergstein Galan ${ }^{1}$, Melina Andrea Formighieri Bertol ${ }^{1}$, Caroline Bortoleto ${ }^{1}$, Natália Santana Siqueira de Lara $^{1}$

${ }^{1}$ Departamento de Medicina Veterinária, Universidade Federal do Paraná

*Autor para correspondência: ana.abreu87@gmail.com

RESUMO: Este trabalho tem por objetivo descrever a coleta de sêmen por estimulação manual do pênis em touro. Foi utilizado um touro da raça Nelore de 96 meses de idade não condicionado para nenhum método de coleta de sêmen. $A$ técnica desenvolvida consistiu em massagem da região perineal até que o animal apresentasse ereção, seguida de pressão na glande até que o animal respondesse com movimentos de fricção. Durante a propulsão, o corpo do pênis foi segurado e pressionado com os dedos, e o copo coletor era posicionado na extremidade livre do pênis para coleta do ejaculado. Após o condicionamento do animal, pelo período de três meses, foram coletados cinco ejaculados em intervalos regulares para avaliação macro e microscópica e $\mathrm{pH}$. Todas as amostras apresentaram aspecto cremoso e branco. O volume médio foi de $6,5 \pm 1,17 \mathrm{~mL}$, a motilidade progressiva média $83 \pm 4,47 \%$, o vigor 5 , o turbilhonamento 5 , o pH 6,05 $\pm 0,09$, a concentração espermática $1,475 \times 10^{9} \pm 0,32 \times 10^{9}$ por $\mathrm{mL}$, o número total de espermatozoides $9,87 \times 10^{9} \pm 3,64 \times 10^{9}$ por ejaculado e a média de espermatozoides anormais totais foi de $9,6 \pm 2,07 \%$. O tempo médio de duração das coletas foi de 176,2 $\pm 12,93$ segs. Concluiu-se que a estimulação manual do pênis pode representar um método promissor para a coleta de sêmen no touro.

Palavras-chave: andrologia; bovino; ejaculado; reprodução.

ABSTRACT: This study describes the semen colletion by manual stimulation of the penis in the bull. A Nellore bull was used unconditioned for any semen collection method. The technique developed consisted of massage of the perineal region until the animal presented erection, followed by pressure in the glans until the animal responded with friction movements. During the propulsion, the penis body was held and pressed with the fingers, and the collection cup was positioned at the free extremity of the penis for collection of the ejaculate. After conditioning the animal, for a period of three months, five ejaculates were collected at regular intervals for macro and microscopic evaluation and $\mathrm{pH}$. All samples had a creamy and white appearance. The mean volume was $6.5 \pm 1.17 \mathrm{~mL}$, the mean progressive motility was $83 \pm 4.47 \%$, the vigor 5 , mass movement 5 , pH $6.05 \pm 0.09$, the spermatic concentration of $1.475 \times 10^{9} \pm 0.32 \times 10^{9}$ per $\mathrm{mL}$, the total number of spermatozoa $9.87 \times 10^{9} \pm 3.64 \times 10^{9}$ per ejaculate and the mean of total abnormal spermatozoa was $9.6 \pm 2.07 \%$. The mean duration of the collection was $176.2 \pm 12.93 \mathrm{sec}$. It is concluded that the manual penis stimulation can represent a promising method for the semen collection in the bull.

Keywords: andrology; bovine; ejaculated; reproduction. 


\section{INTRODUÇÃO}

As técnicas descritas para coleta de sêmen em touros in vivo incluem vagina artificial, eletroejaculação e massagem retal das ampolas do ducto deferente (Sylla et al., 2015), sendo também possível a recuperação de espermatozoides viáveis da cauda de epidídimo, post mortem ou após orquiectomia, utilizando a técnica de fluxo retrógrado (Bertol et al., 2013). A vagina artificial é o método menos estressante e mais fisiológico de coleta de sêmen, sendo a técnica mais representativa, pois simula a monta natural, permitindo a avaliação da libido do macho e conservando as características seminais (Palmer et al., 2013; Sylla et al., 2015). Essa técnica é amplamente utilizada em centrais de inseminação artificial, no entanto pode ser considerada difícil de ser executada, além de demorada e algumas vezes perigosa quando envolve touros potencialmente indóceis, e, portanto, em alguns casos, não passível de uso em condições a campo (Persson et al., 2007). Para Sally et al. (2015), qualquer outro método de coleta para avaliação de sêmen de touros que não permitem a coleta através da vagina artificial é de grande relevância para a bovinocultura de corte.

Em se tratando da eletroejaculação, esse método de coleta não permite a avaliação da libido do reprodutor (Barth et al., 2004). O sêmen coletado por esse método apresenta maior volume quando comparado à vagina artificial, no entanto o número total de espermatozoides não difere entre os ejaculados obtidos em ambas as técnicas (Austin et al., 1961). Além disso, a eletroejaculação é proibida em alguns países, sendo necessária uma técnica alternativa de coleta de sêmen para touros a campo (Persson et al., 2007).

A massagem retal das ampolas dos ductos deferentes é um dos métodos mais simples de coleta de sêmen em bovinos (Palmer et al., 2005). Porém, os ejaculados coletados desta forma apresentam baixa qualidade, baixa concentração espermática e alta contaminação (Ohashi, 2008). Palmer et al. (2005) demonstraram que amostras de sêmen de touro coletadas por massagem das ampolas apresentaram menor porcentagem de motilidade e de espermatozoides vivos, quando comparadas às amostras de sêmen obtidas por eletroejaculação.

A coleta de sêmen por estimulação manual do pênis é o método de eleição para a espécie canina (Kutzler, 2005) e suína (Hancock e Howell, 1959). E esta técnica já foi descrita em outros animais como no garanhão (Crump e Crump, 1989; McDonell e Love, 1990), na zebra (Crump e Crump, 1994) e no morcego (Melville et al., 2008) porém não existem relatos em bovinos, até o momento. $\mathrm{O}$ objetivo deste trabalho é descrever a coleta de sêmen em bovino por estimulação manual do pênis.

\section{MATERIAL E MÉTODOS}

Para a realização deste estudo foi utilizado um touro puro de origem da raça Nelore (Bos taurus indicus), de 96 meses de idade, mantido a campo e utilizado para monta natural. O animal não foi condicionado para nenhuma técnica de coleta de sêmen. Para o desenvolvimento do trabalho, o touro foi transportado e alojado em baia individual no Hospital Veterinário da Universidade Federal do Paraná, onde recebia dieta a base de silagem de milho e feno de azevém, além de água e sal mineral ad libitum. Na primeira fase do experimento, o reprodutor foi submetido ao processo de condicionamento que consistiu na realização de massagem perineal e estimulação manual do pênis duas vezes por semana com intervalos 
regulares, durante três meses consecutivos. Estes procedimentos ocorriam sempre no mesmo horário pela manhã, mesmo ambiente e foram realizados pela mesma pessoa. Ao término de cada sessão o touro recebia uma recompensa (alimento) e, então solto em piquete. $\mathrm{O}$ reprodutor apresentava temperamento dócil, minimizando os riscos para o técnico.

Previamente às coletas de sêmen, o prepúcio era higienizado com ducha de água morna $\left(36^{\circ} \mathrm{C}\right)$ e seco com papel toalha. A coleta pelo método de estimulação manual não foi feita na presença de vaca em estro e não necessitava de contenção do animal. A técnica consistiu em massagem da região perineal (Figura 1A), com movimentos de cima para baixo, até que o animal apresentasse ereção com exposição da extremidade livre do pênis no óstio prepucial. Após a ereção, foi exercida pressão com os dedos na glande (Figura 1B) até que o animal respondesse com movimentos de fricção. No momento da propulsão, o corpo do pênis foi segurado e pressionado com a mão enluvada, para mimetizar a vagina da vaca, e o copo coletor foi posicionado na extremidade livre do pênis para coleta do ejaculado (Figura 1C). O copo coletor foi constituído por um funil de vidro acoplado a um tubo de ensaio esterilizado e aquecido a $36^{\circ} \mathrm{C}$.

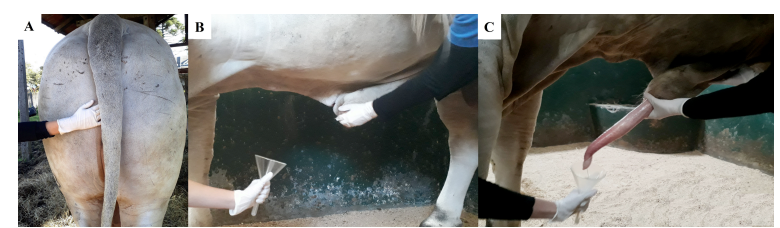

Figura 1 - Técnica de coleta de sêmen por estimulação manual do pênis no touro (A: massagem na região perineal; $B$ : pressão digital na glande; C: coleta do ejaculado no momento da propulsão).

Após o condicionamento do animal foram realizadas duas coletas por semana com intervalos de três dias, durante três semanas consecutivas para avaliação dos ejaculados e para cronometrar a duração da coleta, desde a excitação até a ejaculação. Estas amostras foram acondicionadas em banho-maria a $36^{\circ} \mathrm{C}$ e avaliadas quanto às características macro e microscópicas: aspecto, volume $(\mathrm{mL})$, motilidade progressiva (\%), vigor espermático (1 a 5), turbilhonamento (1 a 5), concentração espermática (por $\mathrm{mL}$ ), número total de espermatozoides (por ejaculado) e morfologia espermática. As avaliações da motilidade, vigor e turbilhonamento foram realizadas de forma subjetiva em microscópio óptico em aumento de 200x, e a concentração espermática determinada por contagem dos espermatozoides em câmara de Neubauer em aumento de 400x. O exame da morfologia espermática foi realizado por meio de esfregaço corado pelo método Cerovsky (CBRA, 2013), sendo feita a contagem diferencial de 200 espermatozoides utilizando aumento de 1000x em óleo de imersão. $\mathrm{O} \mathrm{pH}$ das amostras de sêmen foi determinado com pHmetro.

\section{RESULTADOS}

Decorridos três meses do início do condicionamento, a coleta de sêmen por estimulação manual foi realizada com sucesso. Após esta etapa, as coletas continuaram sendo realizadas duas vezes por semana, algumas vezes por pessoas diferentes. Havia sessões em que ocorria ereção e ejaculação e outras em que a coleta foi improdutiva. Decorrido mais um mês deste trabalho, a coleta de sêmen foi produtiva em $90 \%$ das tentativas, mesmo quando realizada por pessoas diferentes. Os resultados das avaliações dos ejaculados e a duração da coleta pela técnica de estimulação manual do pênis foram descritos na Tabela 1. Todas as amostras apresentaram aspecto cremoso e branco. O volume médio foi 
de 6,5 $\pm 1,17 \mathrm{~mL}$; motilidade progressiva média $83 \pm 4,47 \%$; vigor 5 ; turbilhonamento 5; pH 6,05 $\pm 0,09$; concentração espermática $1,475 \times 10^{9} \pm$ $0,32 \times 10^{9}$ por $\mathrm{ml}$; número total de espermatozoides $9,87 \times 10^{9} \pm 3,64 \times 10^{9}$ por ejaculado e percentual médio de espermatozoides anormais de 9,6 \pm $2,07 \%$. O tempo médio de duração da coleta foi de $176,2 \pm 12,93 \mathrm{seg}$.

Tabela 1 - Resultados das avaliações espermáticas de 5 ejaculados consecutivos coletados pela estimulação manual do pênis em um touro.

\begin{tabular}{|c|c|c|c|c|c|c|c|c|c|c|}
\hline Ejaculado & $\begin{array}{l}\text { Vol } \\
\text { (mL) }\end{array}$ & $\begin{array}{l}\text { Corl } \\
\text { aspecto }\end{array}$ & $\begin{array}{l}\text { Mot } \\
(\%)\end{array}$ & $\begin{array}{l}\text { Vigor } \\
\text { (1 a 5 })\end{array}$ & $\begin{array}{c}\begin{array}{c}\text { Turb } \\
\text { (1 a a })\end{array} \\
\end{array}$ & $\begin{array}{c}\text { Conc } \\
10^{\circ} \mathrm{sptz} / \mathrm{mL}\end{array}$ & $\begin{array}{l}\mathrm{N}^{\circ} \text { total } \\
10^{\circ} \mathrm{sptz}\end{array}$ & $\begin{array}{c}\text { Def } \\
\text { Sptz (\%) }\end{array}$ & $\mathrm{pH}$ & $\begin{array}{c}\begin{array}{c}\text { Tempo } \\
\text { (segundos) }\end{array}\end{array}$ \\
\hline 1 & 5,5 & $\begin{array}{l}\text { brancol } \\
\text { cremoso }\end{array}$ & 80 & 5 & 5 & 1,05 & 5,77 & 8 & 6,10 & 176 \\
\hline 2 & 7,5 & $\begin{array}{l}\text { brancol } \\
\text { cremoso }\end{array}$ & 80 & 5 & 5 & 1,65 & 12,37 & 10 & 6,16 & 195 \\
\hline 3 & 7,5 & $\begin{array}{l}\text { brancol } \\
\text { cremoso }\end{array}$ & 90 & 5 & 5 & 1,8 & 13,5 & 12 & 5,94 & 180 \\
\hline 4 & 7,0 & $\begin{array}{c}\text { brancol } \\
\text { cremoso }\end{array}$ & 80 & 5 & 5 & 1,65 & 11,55 & 7 & 5,99 & 160 \\
\hline 5 & 5,0 & $\begin{array}{l}\text { brancol } \\
\text { cremoso }\end{array}$ & 85 & 5 & 5 & 1,23 & 6,13 & 11 & 6,06 & 170 \\
\hline
\end{tabular}

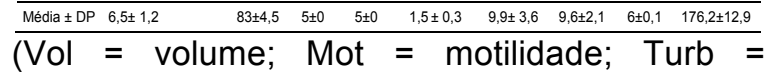
turbilhonamento; Conc sptz/ $\mathrm{ml}=$ concentração de espermatozoides por $\mathrm{ml} ; \mathrm{N}^{\circ}$ total $\mathrm{sptz}=$ número total de espermatozoides no ejaculado).

\section{DISCUSSÃO}

A técnica de coleta de sêmen por estimulação manual apresenta vantagens em relação à vagina artificial e à eletroejaculação. Dentre elas destacam-se a facilidade na execução após o condicionamento do animal, eliminação da necessidade de fêmea em estro e/ou manequim e de contenção do animal (menor estresse), menor custo operacional e instrumental, menor tempo gasto durante 0 procedimento e necessidade de menor número de pessoas para a realização do procedimento, vantagens relevantes para as centrais de reprodução. Além disso, poderia ser usado para reprodutores com problemas locomotores ou neurológicos que apresentam dificuldade, ou incapacidade, para realizar a monta.

Dentre as dificuldades para a realização da técnica estão o temperamento do touro e o tempo relativamente longo para o condicionamento. Da mesma forma, para a coleta de sêmen com a vagina artificial, os machos também necessitam de condicionamento (Palmer, 2005). Outra limitação do método de estimulação manual é o fato de não ser possível avaliar o comportamento sexual do animal durante a cópula, parâmetro importante durante o teste de libido. Por este motivo, a técnica é recomendada para a coleta de sêmen de touros em centrais de coleta de sêmen, e não para avaliação andrológica.

Muito tem sido feito em relação ao bem-estar animal. Existem técnicas alternativas para eletroejaculação, visto que essa técnica de coleta de sêmen ocasiona desconforto aos reprodutores (Filho et al., 2009). Dentre elas, destacam-se a vagina artificial convencional, massagem transretal das ampolas, uso de medicamentos (Palmer, 2005) e a vagina artificial interna (Barth et al., 2004). Em bovinos, a técnica de estimulação manual do pênis pode representar mais uma alternativa para a coleta do sêmen, gerando mínimo ou nenhum estresse ao touro.

Atualmente os programas de melhoramento genético incluem temperamento dentre seus parâmetros de seleção. Busca-se selecionar animais com comportamento dócil, visto que estes representam menores fatores de risco para quem os manuseia e melhor desempenho produtivo (Voisinet, 1997). Em estudo realizado por Figueiredo et al. (2005), com 5754 animais da raça Nelore, aproximadamente $\quad 50 \% \quad$ foram classificados como levemente reativos, $29 \%$ como dóceis e $4 \%$ como muito dóceis. Deve ser levado em consideração que os touros a serem condicionados para a realização da coleta de sêmen por estimulação 
manual do pênis sejam selecionados de acordo com o temperamento, preconizando a docilidade.

Diversos

pesquisadores

demonstraram a influência do método de coleta de sêmen sobre as características do ejaculado. Leon et al. (1991), analisaram 51 ejaculados coletados por vagina artificial, provenientes de 20 touros, e encontraram os seguintes resultados: volume médio $5,8 \mathrm{~mL}$, motilidade progressiva média $80 \%, \mathrm{pH} 6,96$ e concentração espermática $1,051 \times 10^{9}$ por $\mathrm{mL}$. Analisando esses dados comparativamente, observamos no presente trabalho valores médios de volume e concentração espermática superiores em relação à vagina artificial, pois resultam em um maior número total de espermatozoides por ejaculado, e consequentemente mais doses inseminantes, uma vez que a motilidade progressiva foi semelhante. Os valores baixos de $\mathrm{pH}$ do sêmen coletado pela técnica proposta podem estar relacionados à dieta ácida a base de silagem de milho, uma vez que Leon et al. (1991) não especificaram a alimentação fornecida aos animais. A avaliação da morfologia espermática encontrou-se dentro dos valores considerados normais para a espécie (CBRA, 2013). Persson et al. (2006) analisaram 47 ejaculados de 52 touros obtidos pela massagem transretal das ampolas os quais apresentaram volume médio de $3,2 \mathrm{~mL}$, motilidade progressiva média $43,5 \%$ e concentração espermática média de $201,9 \times 10^{6}$ por $\mathrm{mL}$. Comparando novamente com os resultados do presente trabalho, os parâmetros espermáticos obtidos através da coleta de sêmen pela estimulação manual do pênis foram superiores aos relatados anteriormente.

\section{CONCLUSÃO}

A estimulação manual do pênis representa um método promissor para a coleta de sêmen no touro, sendo necessários mais estudos para comprovar sua repetibilidade.

\section{REFERÊNCIAS}

AUSTIN, J.W.; HUPP, E.W.; MURPHREE, R.L. Comparison of quality of bull semen collected in the artificial vagina and by electroejaculation. Journal of Dairy Science, v.44, p. 2292- 2297, 1961.

BARTH, A.D.; ARTEAGA, A.A.; BRITO, L.F.C. et al. Use of internal artificial vaginas for breeding soundness evaluation in range bulls: an alternative for electroejaculation allowing observation of sex drive and mating ability. Animal Reproduction Science, v.84, p.315-325, 2004.

BERTOL, M. A. F.; WEISS, R. R. ; SOCCOL, V. T. et al. Viability of Bull Spermatozoa Collected from the Epididymis Stored at $18-20^{\circ} \mathrm{C}$. Brazilian Archives of Biology and Technology, v. 56, p. 777-783, 2013.

COLÉGIO BRASILEIRO DE REPRODUÇÃO ANIMAL - CBRA. Manual para exame andrológico e avaliação de sêmen animal. 3.ed. Belo Horizonte: CBRA, 2013.

CRUMP, J.; CRUMP, J. Stallion ejaculation induced by manual stimulation of the penis. Theriogenology, v.31, n.2, p.341-346, 1989.

CRUMP, J.P; CRUMP, J.W. Manual semen collection from a grevy's zebra stallion (Equus grevyi), onset of sperm production, semen characteristics, and cryopreservation of semen, with a comparison to the sperm production from a grant's zebra stallion (Equus 
burchelli boehmi). Theriogenology, v.41, p.1011-1021, 1994.

FIGUEIREDO, L.G.G.; ELER, J.P.; MOURÃO, G.B. et al. Análise genética do temperamento em uma população da raça Nelore. Livestock Research for Rural Development, v. 17, n. 17, p. 1-7, 2005.

FILHO W. C. M.; FERREIRA, J. C. P.; FUGIHARA, C. J. Indicadores de bemestar em touros submetidos a colheita de sêmen por eletroejaculação. Veterinária e Zootecnia, v.16, n1, p.5263, 2009.

HANCOCK, J.L.; HOWELL, G.J.R. The collection of boar semen. The Veterinary Record, v.71, p.664-665, 1959.

KUTZLER, M.A. Semen collection in the dog. Theriogenology, v.64, p.747-754, 2005.

LEON, H.; PORRAS, A.A.; GALINA, C.S. et al. Effect of collection method on semen characteristics of zebu and european type cattle in the tropics. Theriogenology, v.36, p.349-355, 1991.

McDONNELL, S.M.; LOVE, C.C. Manual stimulation collection of semen from sallions: training time, sexual behavior and semen. Theriogenology, v.33, n.6, p.1201-1210, 1990.

MELVILLE, D.F.; CRICHTON, E.G.; PATERSON-WIMBERLEY, $T$. et al. Collection of semen by manual stimulation and ejaculate characteristics of the black flying-fox (Pteropus alecto). Zoo Biology. v.27, p. 159-164, 2008.

PALMER, C.W. Welfare aspects of theriogenology: Investigating alternatives to electroejaculation of bulls. Theriogenology, v. 64, p.469-479, 2005.

PALMER, C.W.; BRITO, L.F.C.; ARTEAGA, A.A. et al. Comparison of electroejaculation and transrectal massage for semen collection in range and yearling feedlot beef bulls. Animal Reproduction Science, v. 87, p. 25-31, 2005.

PALMER, C.W.; PERSSON, Y.; SÖDERQUIST, L. Classification of the potential breeding ability of range beef bulls based on semen quality parameters in samples collected by transrectal massaged a comparison of the Swedish and Canadian systems. Animal Reproduction Science, v.140, p.124-30, 2013.

PERSSON, Y.; MCGOWAN, M.; SODERQUIST, L. Comparison between the sperm morphology in semen samples obtained from yearling beef bulls by transrectal massage of the ampullae and cauda epididymal dissection. Reproduction Domestics Animals, v.41, p.233-237, 2006.

PERSSON, Y.; STRID, G.; HAARD, M.; SODERQUIST, L. Comparison of semen samples collected from beef bulls by transrectal massage or artificial vagina. The Veterinary Record, v.161, p.662663, 2007.

SYLLA, L.; PALOMBI, C.; STRADAIOLI, G.; VAGNILUCA, A.; MONACI, M. Effect of semen collection by transrectal massage of accessory sexual glands or artificial vagina on the outcome of breeding soundness examinations of Italian yearling beef bulls. Theriogenology, v.83, p.779-785, 2015.

VOISINET, B.D.; GRANDIN, T.; TATUM J.D. et al. Feedlot cattle with calm temperaments have higher average daily gains than cattle with excitable temperaments. Journal Animal Science, v. 75, p. 892- 896, 1997. 